\title{
Frequent recombination of pneumococcal capsule highlights future risks of emergence of novel serotypes.
}

Rafał J. Mostowy ${ }^{1, \dagger}$, Nicholas J. Croucher ${ }^{1}$, Nicola De Maio ${ }^{2,3}$, Claire Chewapreecha ${ }^{4}$, Susannah J. Salter $^{5}$, Paul Turner ${ }^{6,7}$, David M. Aanensen ${ }^{1,8}$, Stephen D. Bentley ${ }^{5}$, Xavier Didelot ${ }^{1}$, Christophe Fraser $^{1,9}$

1) Department of Infectious Disease Epidemiology, School of Public Health, Imperial College London, W2 1PG

London, UK

2) Nuffield Department of Medicine, University of Oxford, Oxford, UK

3) Institute for Emerging Infections, Oxford Martin School, Oxford, UK

4) Department of Medicine, University of Cambridge, Cambridge, UK

5) The Wellcome Trust Sanger Institute, Wellcome Trust Genome Campus, Hinxton, Cambridge CB10 1SA, UK

6) Centre for Tropical Medicine and Global Health, University of Oxford, Oxford, UK

7) Cambodia-Oxford Medical Research Unit, Angkor Hospital for Children, Siem Reap, Cambodia

8) Centre for Genomic Pathogen Surveillance, Wellcome Genome Campus, Hinxton, Cambridge CB10 1SA, UK

9) Oxford Big Data Institute, Li Ka Shing Centre for Health Information and Discovery, Nuffield Department of Medicine, University of Oxford, Oxford, UK

$\dagger$ Corresponding author: r.mostowy@imperial.ac.uk 


\begin{abstract}
Capsular diversity of Streptococcus pneumoniae constitutes a major obstacle in eliminating the pneumococcal disease. Such diversity is genetically encoded by almost 100 variants of the capsule polysaccharide locus $(c p s)$. However, the evolutionary dynamics of the capsule - the target of the currently used vaccines remains not fully understood. Here, using genetic data from 4,469 bacterial isolates, we found cps to be an evolutionary hotspot with elevated substitution and recombination rates. These rates were a consequence of altered selection at this locus, supporting the hypothesis that the capsule has an increased potential to generate novel diversity compared to the rest of the genome. Analysis of twelve serogroups revealed their complex evolutionary history, which was principally driven by recombination with other serogroups and other streptococci. We observed significant variation in recombination rates between different serogroups. This variation could only be partially explained by the lineage-specific recombination rate, the remaining factors being likely driven by serogroup-specific ecology and epidemiology. Finally, we discovered two previously unobserved mosaic serotypes in the densely sampled collection from Mae La, Thailand, here termed $10 \mathrm{X}$ and 21X. Our results thus emphasise the strong adaptive potential of the bacterium by its ability to generate novel serotypes by recombination.
\end{abstract}

\title{
Introduction
}

Streptococcus pneumoniae is a human bacterial commensal and pathogen, estimated to be the cause of death in over 500,000 children under 5 years of age each year worldwide (WHO, 2012). The bacterium's capacity to cause disease is associated with its possession of several virulence factors, of which the most important is the surface polysaccharide capsule (Briles et al., 1992; Morona et al., 2004; Kadioglu et al., 2008; Hyams et al., 2010). The polysaccharide diversity constitutes a major challenge for eliminating pneumococcal disease. Being exposed to antibody binding as the outermost layer of the bacterium, the capsule is the target of all licensed pneumococcal vaccines. The most commonly used conjugate vaccines currently target eleven or thirteen of the most common capsular types (serotypes). However, as of today almost 100 distinct serotypes have been described and recognised. Each serotype has a unique, experimentally confirmed serological profile (Henrichsen, 1995), and for many of them the biochemical structure is known (Geno et al., 2015).

Systematic genetic sequencing revealed that the diversity of the capsule locus, cps, alone forms a repertoire of almost 2,000 unique genes (Bentley et al., 2006). These genes are divided based on their functions and form three major groups (Yother, 2011; Geno et al., 2015). The first group is located upstream of the locus and consists of modulatory cpsABCD genes (wzg, wzh, wzd, wze), 
which are common to almost all serotypes. The second group are serotype-specific genes (i.e., glycosyltrasferases) with polymer-specific functions, and these define a serotype. Finally, many serotypes carry sugar-synthesis genes needed for capsule production (e.g., rhamnose genes). Comparison of the genetic content of different serotypes demonstrated that capsular-gene acquisition and loss had been the underlying cause of emergence of many serotypes (Aanensen et al., 2007; Mavroidi et al., 2007). This is not surprising as the pneumococcus is known to undergo frequent recombination (Feil et al., 2000; Henriques-Normark et al., 2008; Vos and Didelot, 2009), and the capsular locus was shown to be a recombination hotspot (Croucher et al., 2011; Chewapreecha et al., 2014). Furthermore, we know from previous studies that the extent of within-serotype diversity is under-appreciated, with many hybrid serotypes circulating in the population (Salter et al., 2012; van Tonder et al., 2016). However, the evolutionary dynamics, and hence the full adaptive potential of pneumococcal capsular polysaccharides, are not well understood.

The aim of this study was to gain a high-resolution view of the evolution of capsular polysaccharides in S. pneumoniae. In particular, we wanted to infer the rates of evolution and recombination within the capsular locus, compare these parameters between different serogroups, and compare the relationship between evolution affecting capsular genes and that affecting the remainder of the genome. To this end, we analysed capsular diversity in a collection of 4,469 bacterial isolates form several different studies. Our approach allowed us to observe the evolution of the pneumococcus from the point of view of the capsule itself, subdivided in major serotypes and serogroups, with the tree showing the evolution of the capsular locus and the tips of the tree containing the information about how the capsule changes between different genomic backgrounds. By disentangling horizontal from vertical genetic changes, we gained insight into the timescales of diversification and recombination in capsular genes. This approach brings novel qualitative and quantitative insight into the evolution of serotypes, the principal target of current vaccines.

\section{Results}

\section{Species-wide serotype diversity}

To study the evolution of the capsular locus, we analysed several collections of pneumococcal isolates including two large carriage cohorts from MaeLa, Thailand (Chewapreecha et al., 2014) and Massachusetts (Croucher et al., 2013); three widespread lineages, PMEN1, PMEN2 and PMEN14 (Croucher et al., 2011, 2014b,a); capsular reference collection (Bentley et al., 2006); Dutch isolates from invasive disease (Elberse et al., 2011); and publically available reference genomes the European 
Nucleotide Archive (ENA). This gave a total number of 4,469 isolates from 29 countries and 5 continents, as shown in Figure 1A. Illumina-sequenced isolates (96\%) were reassembled using a novel pipeline (see Text S1). In total, we obtained 3,813 full capsular sequences, which were serotyped in silico (see Methods and Table S1). Figure 1B shows the observed serotype distribution, with $47 \%$ of the identified capsular sequences being serotypes targeted by the seven-valent pneumoccal conjugate vaccine (PCV7) and $59 \%$ being serotypes targeted by the more recent thirteen-valent vaccine (PCV13). Altogether we identified 96 reference serotypes consisting of 254 glycosyltransferase gene families (henceforth referred to as capsular-specific genes), amongst which we discovered two previously unseen, putative serotypes, here referred to as serotype 10X and serotype $21 \mathrm{X}$, respectively (see Figures S1/S2). Putative serotype 10X was found in five isolates from MaeLa and originally classified as 10B, 10F or 33B. Accordingly, the genetic structure suggests that it is a mosaic of 10C or $10 \mathrm{~F}$ with another serotype, possibly $35 \mathrm{~B}$. Putative serotype $21 \mathrm{X}$ was found in three isolates from MaeLa, and its genetic structure suggests that it arose as a recombination between $6 \mathrm{C} / 6 \mathrm{D}$ and 39 . Indeed, $21 \mathrm{X}$ reacted to antiserum which covers serotypes 33 and 39 , and the presence of the novel, functional polysaccharide was reconfirmed (see Text S1).

To visualise capsular diversity within the pneumococcus, we generated a network with nodes represented by reference sequences and edges linking serotypes with similar capsule-specific gene content. Fig. 1C shows this similarity network for two different thresholds, conservative and liberal, with two corresponding edge types. We see that serotype clusters in the conservative network (henceforth referred to as genetic serogroups) are often congruent with phenotypic serogroups. Nevertheless, there are exceptions: for example serotype 16F clusters with serogroup 28 with conservative threshold, but does not cluster with 16A even with liberal threshold. These observations are consistent with earlier findings and highlight the complexity of the polysaccharide genotypephenotype map (Aanensen et al., 2007; Mavroidi et al., 2007). This approach also allows identification of mosaic triplets (nodes connecting other groups), which denote potential introgressive descents (Bapteste et al., 2012). In this way one can quickly identify some of the mosaic serotypes, for example $18 \mathrm{~F}$ (shares $w c x M$ gene with $18 \mathrm{~A} / 18 \mathrm{~B} / 18 \mathrm{C}$ and $28 \mathrm{~A} / 28 \mathrm{~F} / 16 \mathrm{~F}$ ) or $22 \mathrm{~A}$ (shares $w c w C$ gene with $22 \mathrm{~F}$ and $7 \mathrm{~A} / 7 \mathrm{~F})$. Furthermore, the largest connected component, which includes serogroups $10,33,34,35$ and others, is the most mosaic group of serotypes. Indeed, a closer look suggests that its members consist of the most interconnected serotypes (see Fig. S3). Therefore, we can conclude that many serotypes are highly mosaic in nature and their evolution was likely driven by horizontal transfer of DNA. 


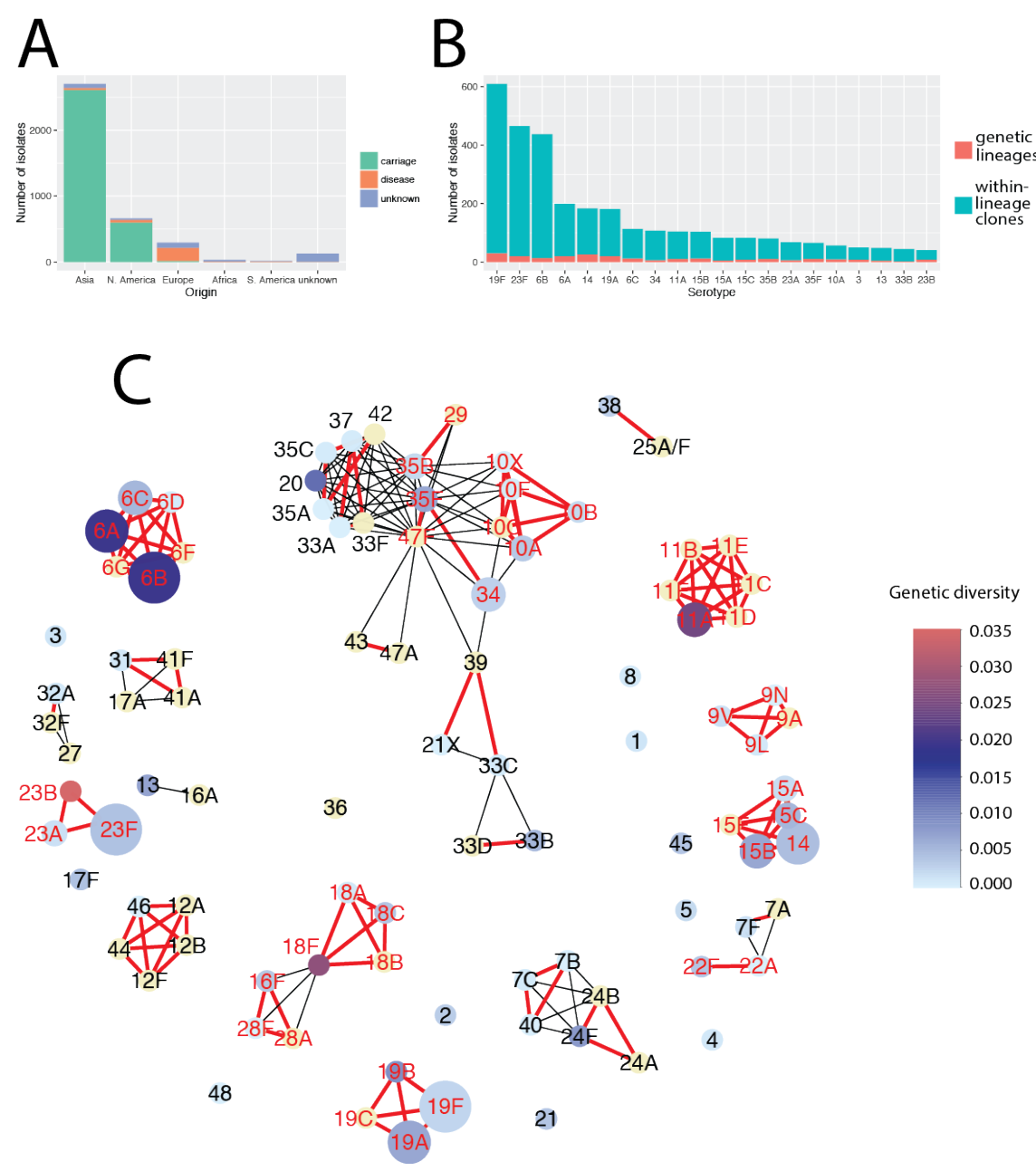

Figure 1: Properties of the dataset. (A) The distribution of the number of isolates stratified by geographical location and the source of isolation (carriage, disease or unknown). (B) The distribution of the number of serotypes. Red bars show the number of genetic serotype lineages, defined by clusters of sequences which can be connected by linking all pairs which differ by two substitutions or less. Blue bars show the remaining count such that the total height equals the full sample size. (C) A diversity network, where each node is represented by a reference sequence and an edge links two nodes if they are similar, i.e., share a minimum proportion $x$ of the homologies. Red, bold edges show the conservative network where the minimum similarity was defined as sharing $x=0.58$ of homologies. Black edges show the additional connections obtained in a liberal network, where the minimum similarity was defined as sharing $x=0.36$ of the homologies. The size of each node reflects the full sample size, and the colour shows the within-serotype capsular genetic diversity for all non-identical isolates measured using the mean pairwise Kimura K80 distance (full diversity distribution is shown in Figure S4). Red labels of serotype nodes denote genetic serogroups which are analysed in detail below. 


\section{Recombination drives emergence of serotypes}

We next investigated the diversification of different serogroups into serotypes. To this end, we focused on the genetic serogroups defined by red edges in Fig. 1C (nodes marked with red labels). As these groups share a large majority of their genetic content, the traditional population genetic methods can be applied to infer the basic evolutionary parameters. We analysed twelve most numerous and diverse serogroups, here referred to as serogroups: $6,19,23,14 / 15,18,10,11$, 9, 34/35, 16/28, 29/35 and 22 (see Fig. S4 for diversity distribution). In brief, sequences from each group were aligned and the population genetic structure was analysed, with recombinations within each sub-population identified using two different methods (Falush et al., 2003; Croucher et al., 2015a). The recombinant fragments were then removed from the alignment and the resulting clonal alignment was used to construct a tree of the serogroup, with recombinations mapped onto the tree. Figure 2 summarises the inferred model of evolution for the four most common serogroups, 6, 19, 23 and 14/15. The details of the analysis for all serogroups are given in Supplementary Text S1 and Figs S15-S38. Here we briefly summarise the main findings.

\begin{tabular}{cccc} 
serogroup & serotype(s) & gene(s) affected & likely source \\
\hline 6 & $6 \mathrm{C}$ & $w c i N$ & unknown (homolog in 21X) \\
6 & $6 \mathrm{D}$ & wciP, wzy, wzx & $6 \mathrm{~B}-\mathrm{II}$ \\
19 & $19 \mathrm{~B} / 19 \mathrm{C}$ & $w c h U$ & S. mitis \\
10 & $10 \mathrm{C}$ & $w c r W$ & $10 \mathrm{~A}$ \\
$34 / 35$ & $47 \mathrm{~F}$ & $w h a I$ & $47 \mathrm{~A}$ \\
$16 / 28$ & $28 \mathrm{~A}$ & $w c i U$ & unknown \\
22 & $22 \mathrm{~A} / 22 \mathrm{~F}$ & $w c w C$ & unknown
\end{tabular}

Table 1: Direct evidence for emergence of serotypes by recombination. The table summarises the cases in recombinations directly detected in our approach were associated with the emergence of new serotypes. In some cases the direction of recombination could not be established; in this case multiple serotypes were given in the second column. Details of the analysis are given in Text S1.

First, the critical event in the emergence of at least seven serotypes was a recombination importing extensive genetic diversity, as indicated by the causative change being associated with a cluster of polymorphisms on the ancestral branches of these serotypes. In four of these cases, the source could be identified (see Table 1 and Text S1). Second, in serogroup 19 we hypothesise that the 19B/19C clade arose by arose by recombination of $19 \mathrm{~F}$ with $S$. mitis, and in serogroup 23 that serotype $23 \mathrm{~A}$ emerged as a recombination of $23 \mathrm{~F}$ and a capsule of an unknown source. Third, we detected many recombinations which did not change serotype but sometimes produced mosaic isolates, for example 19A/19F mosaic, 6B-I/6B-II mosaic or 23B-mosaic. Fourth, population genetic 
structure of the common wz-genes shows presence of many older, undetected recombinations (see Fig. S5). Finally, in many cases we observed that a simple model of gene gain and loss cannot explain the observed patterns of diversity. In particular, we found serotypes $6 \mathrm{~A} / 6 \mathrm{~B}, 15 \mathrm{~B} / 15 \mathrm{C}$ and $18 \mathrm{~B} / 18 \mathrm{C}$ to have emerged on multiple independent occasions, some of which were due to homologous recombination (though in serogroup 15 it could be due to intragenomic recombinations; see Fig. 2 and Text S1). Hence the evolutionary history of these serotypes is a complex story of repeated recombinations of differing phenotypic consequences. 
A

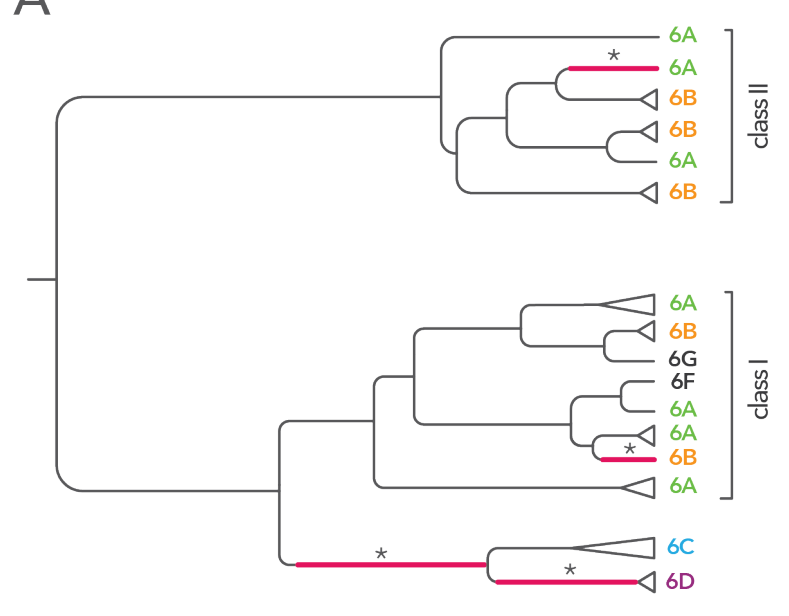

B

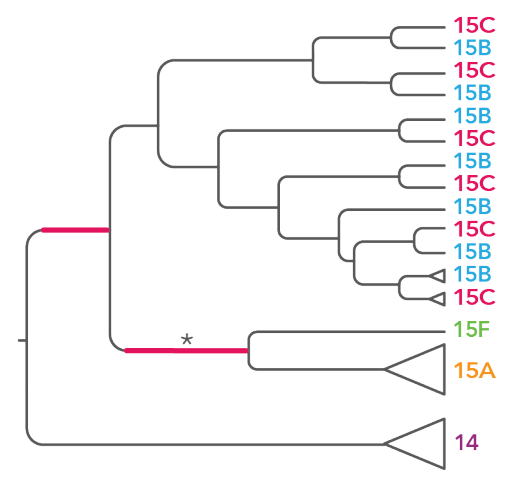

C
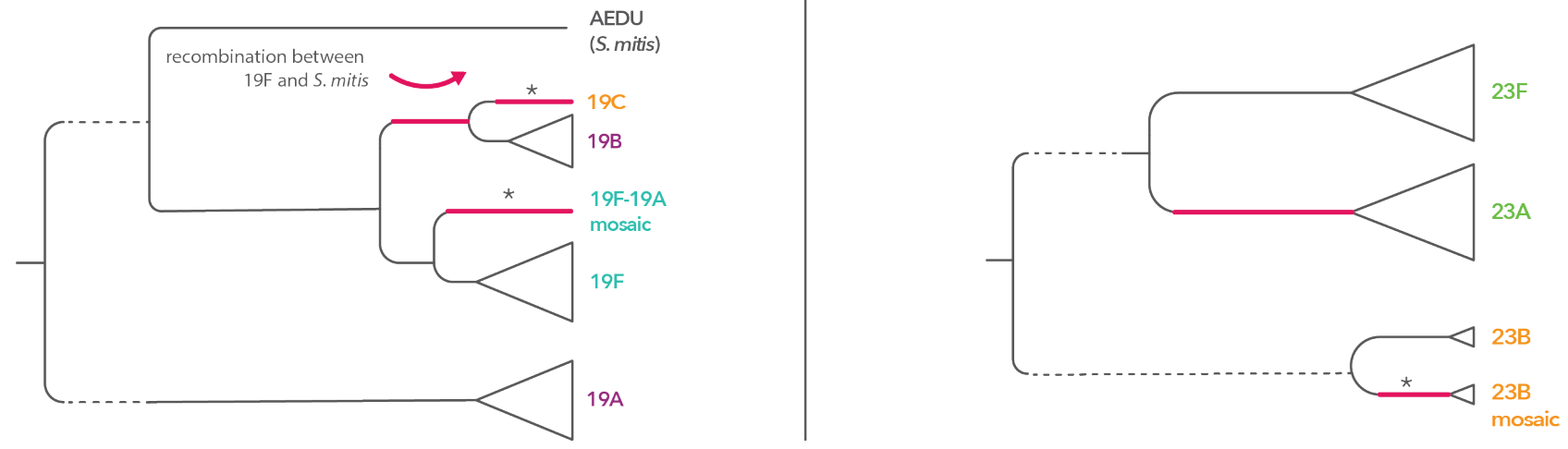

Figure 2: Evolution of the four most common serogroups. Schematic dendogram based on the inferred clonal trees is shown for the four most common serogroups in this study, serogroup 6 (panel A), serogroup 14/15 (panel B), serogroup 19 (panel C), and serogroup 23 (panel D). Branches where recombinations occurred are coloured red, with a star sign marking those branches where there was statistical support for the recombination (using Structure or Gubbins). Clonal uncertainty due to the suggested model is reflected by dashed branches.

\section{Molecular clock of the capsule}

We next wanted to learn about the timescales of the evolutionary process within the capsule. Unfortunately, the serogroup alignments did not have enough temporal signal to robustly infer the substitution rate of the capsular locus. Therefore, we used whole-genome collections of three 
lineages PMEN1, PMEN2 and PMEN14, respectively (Croucher et al., 2011, 2014b,a). We inferred alignments with recombinations removed using the software Gubbins (Croucher et al., 2015a). For each lineage, we simultaneously fitted two separate clock models, one to the entire alignment with the capsule removed, and the other one to the capsule only, defined by coordinates of $\operatorname{dex} B$ and aliA genes (see Methods), all using BEAST2 (Bouckaert et al., 2014). Results are displayed in Figure $3 \mathrm{~A}$ and show that in all three lineages we observed a roughly 2.5 times higher clock rate at the capsular locus than in the rest of the genome. The distribution of SNPs across capsular genes (see Fig. S6) suggests no bias at transposable elements, which can sometimes produce false-positive substitutions due to their repetitive nature. We can thus conclude that the observed substitution rate is not an artefact of data assembly. To investigate whether the increased substitution rate at the capsule is due to varying rates of selection between different proteins, we estimated the $\mathrm{dN} / \mathrm{dS}$ ratio using the CODEML approach (Yang, 2007) for the capsule versus all other genes (see Methods). Results are shown in Figure 3B and suggest that the capsule has a higher proportion of non-synonymous substitutions compared to the rest of the genome. It is unclear whether the increased $\mathrm{dN} / \mathrm{dS}$ is due to positive, diversifying selection or relaxed purifying selection.

To examine whether there may have been any undetected recombinations in the capsule, which in turn could explain the elevated substitution rate, we tested for the levels of homoplasies using phitest (Bruen et al., 2006). In all three cases we found non-significant results $(p=0.1, p=1$ and $p=0.1$, for PMEN1, PMEN2 and PMEN14, respectively). A closer examination also does not point to a substantial impact of undetected recombinations. In PMEN1 and PMEN2 the proportion of homoplasic substitutions was only $6 \%$ and $7 \%$, respectively, and in PMEN2 we would not expect a substantial impact as many representatives of this clone had their competence turned off by a phage insertion. In PMEN14 the proportion of homoplasic substitutions was $21 \%$, but in this case the higher number is likely due to selection: of 48 independently occurring SNPs across all capsular coding regions, 38 were located within two genes, wzd and wze (see Fig. S5). The estimated $\mathrm{dN} / \mathrm{dS}$ in those two genes was many times higher than across all capsule: 1.25 (95\% CIs: 0.70-2.65) compared to 0.057 (0.049-0.066). Thus, even though the impact of recombination on the elevated substitution rate cannot be excluded, the higher molecular clock observed at the capsule is most likely explained by an impact of selection. 


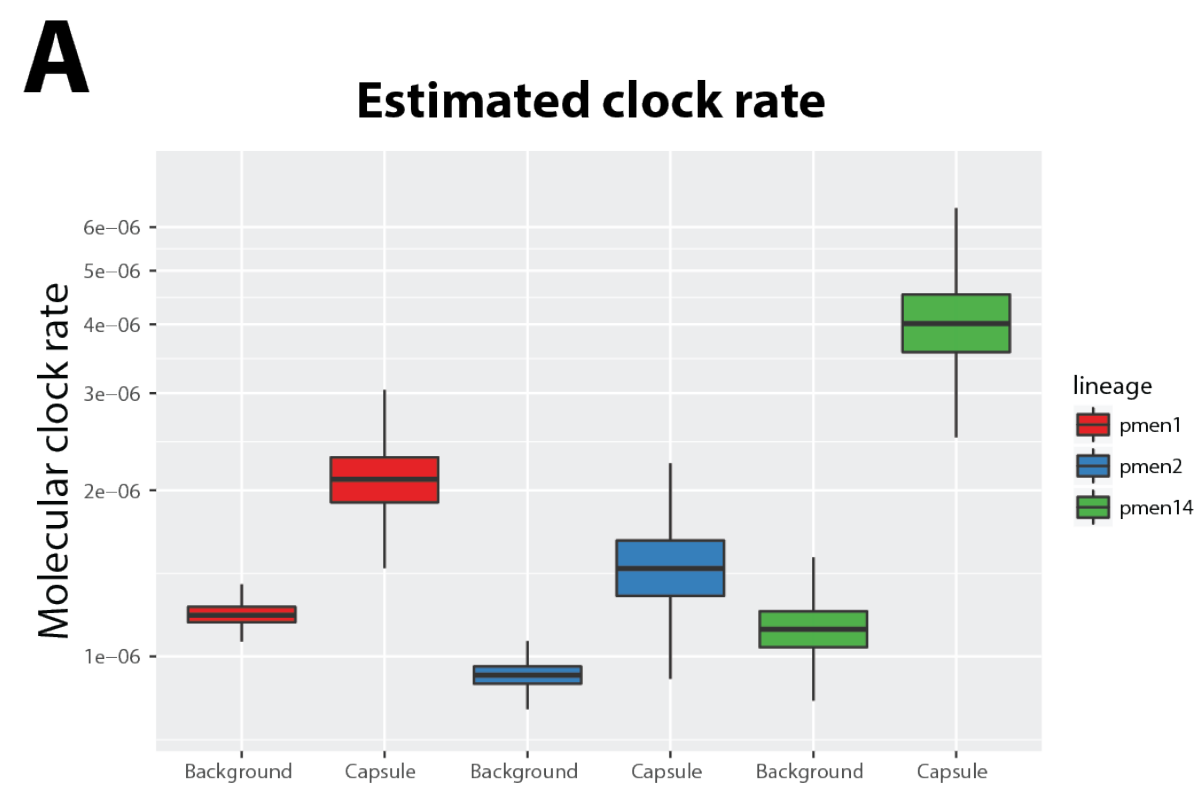

B

\section{Measure of selection}

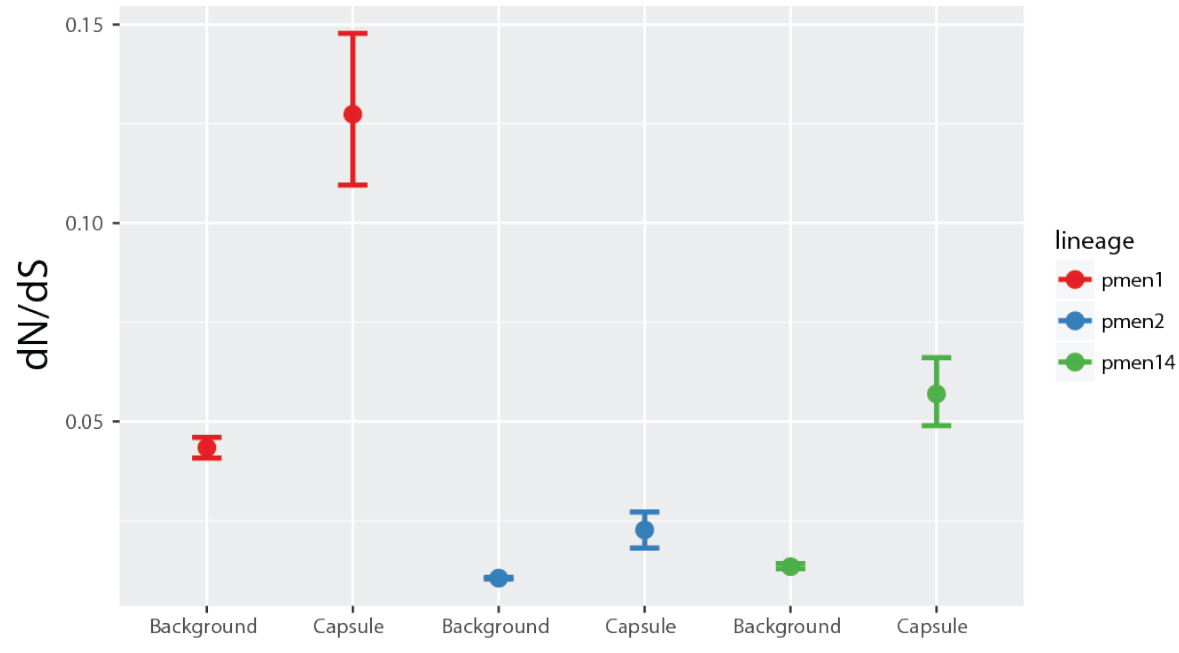

Figure 3: The molecular clock rate of the capsular locus versus background in three different lineages, PMEN1, PMEN2 and PMEN14. (A) The bar plots show the inferred molecular clock rate of the whole-genome alignment with the capsule removed ("Background"), and to the capsule-only alignment ("Capsule"). (B) Comparison of the selective pressures exerted on the background vs. capsule as inferred by $\mathrm{dN} / \mathrm{dS}$. 


\section{Variability in recombination rates between serogroups}

Using the capsular clock rate estimated for PMEN collections and the sampling dates obtained for the isolates used in this study, we next estimated the branch lengths of the ML trees for each of the serogroups. This also allowed us to infer the divergence times of the capsular clonal tree (shown in Figs. S7-S10) with the times of occurrence of corresponding recombination events, as well as to estimate the recombination rate for each serogroup in more intuitive units. Figure 4A shows the comparison of the obtained recombination rate estimates for the 12 examined serogroups. The results demonstrate a significant heterogeneity in recombination rates across all serogroups (oneway anova, $p<10^{-16}$ ), though pairwise differences were often statistically non-significant. To test whether sampling can explain the observed variance in recombination rates, we examined associations between the rates and five measures of sampling: number of isolates, number of nonidentical isolates, clonal diversity, number of countries in which each serogroup was sampled and number of clonal complexes (see Table S1 in Text S1). We did not find a significant association between the recombination rate and any of the quantities (Spearman rank test, $p>0.05$ in all cases). As corresponds to theoretical expectations (see Fig. S11), we conclude that sampling cannot explain the observed variation in recombination rates.

The second hypothesis which could explain the variation in recombination rates is the selective pressure to preserve the polysaccharide structure by maintaining capsular genetics. That such selection might act is already suggested by the recombination heatmap across the capsular locus (Fig. 4B): we found the recombination frequency higher at the flanks of the capsule, namely within those genes which are common to many serotypes. One potential explanation for this is that those regions are less likely to disrupt the capsule-specific polysaccharide structure and are thus observed more often due to negative purifying selection. However, more frequently observed recombinations are also expected to occur at a higher rate at relatively conserved sites due to a higher number of possible donor-recipient pairs in the homologous recombination process, which requires sequencesimilarity at flanking regions. To circumvent this problem, we used a whole-genome lineage-bylineage approach in all used data collections to detect recombinations with breakpoints outside the analysed capsular region and thus invisible in our alignments (see Methods). By analysing only lineages which were at least $95 \%$ of a single serogroup, we could directly compare the frequency of (i) non-capsular homologous recombinations, (ii) all recombinations affecting the capsule and (iii) recombinations contained within the capsule. Fig. 4C shows the comparison of the mean withincapsule recombination rate (left) and the mean total capsule recombination rate (right). Under a neutral model we would expect $49.6 \%$ of all capsular recombinations to be contained within 
the capsular locus (see Text S1 for full calculation). Comparison of the recombination rate ratios revealed that only serogroup 6 was found to lie within that range $(0.500$, CI $0.136-0.733)$, while for the other three serogroups we found less within-capsule recombinations than expected (serogroup 19: 0.313, CI 0.177 - 0.443; serogroup 23: 0.258 , CI $0.161-0.357$; serogroup 14/15: 0 ). These results suggest that within-capsular recombinations may be under different selective pressures in different serogroups (see Discussion).

Another hypothesis that could explain the variation in recombination rates is the background recombination rate, which is known to vary between lineages (Croucher et al., 2013). To investigate this we asked whether the genomic recombination rate is a good predictor of the capsule recombination rate (Fig. 4D). We found that the former explains roughly half of the variance in recombination rates between serotypes (linear model, $R^{2}=0.47, p<0.01$ ). Finally, we found no significant relation between the mean serogroup capsule size, as measured experimentally (Weinberger et al., 2009), and the estimated recombination rate (see Fig. S12). As capsules are known to constitute a physical barrier to incoming transformation events (Schaffner et al., 2014), this result suggests that the variation in observed recombination rates cannot be explained by raw transformation rates, but rather reflects the differential effect of selection on the capsular locus. 


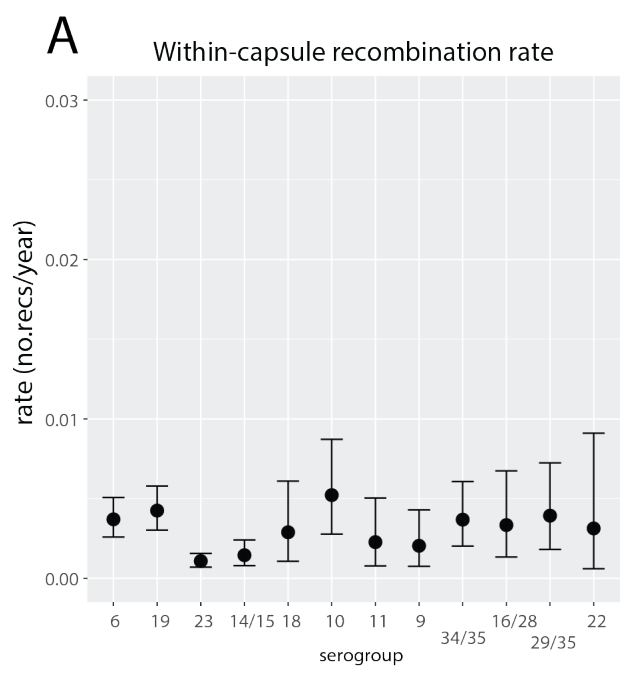

B Capsule-recombination heatmap
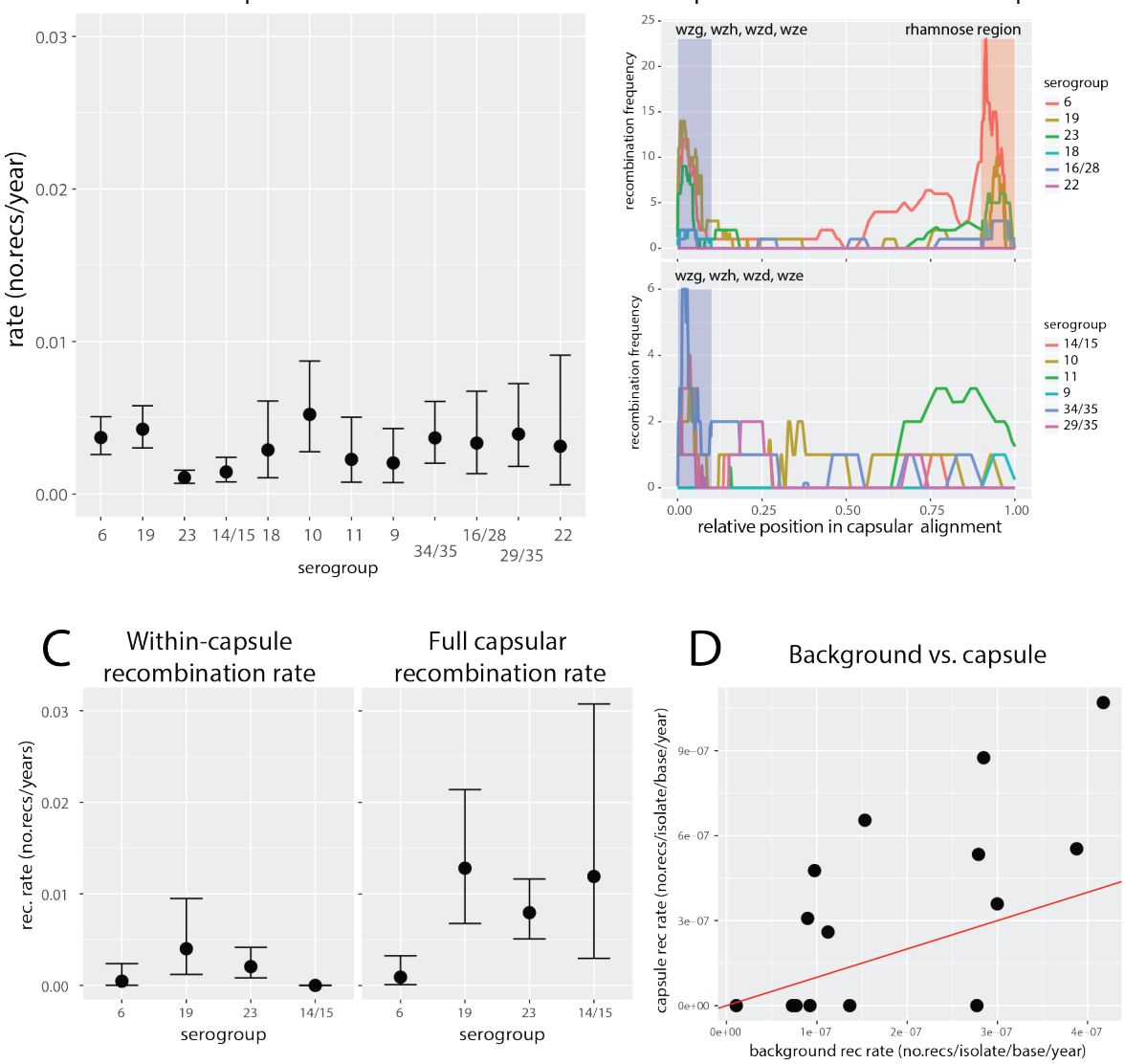

Figure 4: Recombination rates within the capsular locus. (A) Recombination rates estimated for the 12 serogroups used in this study, with the relevant $95 \%$ confidence intervals. By definition these rates do not include long, capsule-spanning recombinations which are invisible from the point of view of capsular alignment. (B) Recombination heatmap at the capsular locus, with different colours showing number of recombinations for different serogroups, with rhamnose genes (top) and without rhamnose genes (bottom). Recombination positions were normalised such that total alignment length was 1 in all serogroups. Additionally, for the sake of comparison, the upstream $w g$-region (blue) and the rhamnose region (red) were normalised to $10 \%$ of the length each. (C) Recombination frequency measured using whole-genome approach, with capsule-contained events (left) vs. all capsule events (right). (D) Recombination rate at the genomic background (excluding events at the capsule) versus recombination rate of events affecting the capsule, estimated from whole-genome alignments. The rates were normalised per base using mean alignment lengths of whole-genome and capsule, respectively. The $y=x$ line is shown in red. 


\section{Origin of capsular recombinations}

We next investigated the origin of the identified recombination events. To this end, we used BLAST to identify close hits (defined by min. $90 \%$ identity; see Methods) with multiple hits assigned a proportionally lower weight; otherwise the origin was considered unknown. We also included a set of 50 S. mitis sequences mentioned in Table S1. Fig. 5A shows a recombination flow diagram, namely a directed network with arrows indicating the direction of recombination between different serogroups. We identified potential source for $91 \%$ of recombinations. It is unclear whether the remaining recombinations descended from the same or other bacterial species. However, we would not expect to find many cases of inter-species recombinations for several different reasons, including biological ones (stronger purifying selection of more diverse imports) and methodological ones (under-sequenced diversity of non-pneumococcal streptococci). Nevertheless, in the case of serogroup 19 we found four recombinations with close homology to S. mitis, and in serotype 14 one recombination with close homology to $S$. oralis. We also found that more recombinations originated in other serogroups compared to same serogroup (156 vs. 105), consistent with the observation in that most recombinations are found in genes common in other serotypes (cf. Fig. $4 \mathrm{C})$. To explore this dependence for each serogroup, we quantified the number of recombinations originating in the same ("self") versus in different ("non-self") serogroup (Figure 5B). We found that most serogroups have more non-self recombinations, but in three serogroups $(6,10$ and 11) the majority of recombinations originated in the same serogroup. (The results were qualitatively similar when considering only capsule-specific recombinations.) These results could explain a lower within-capsule recombination rate in serogroups 19, 23 and 14/15 than expected: recombinations originating in other serogroups are more likely to disrupt the capsule, and hence be selected against. 

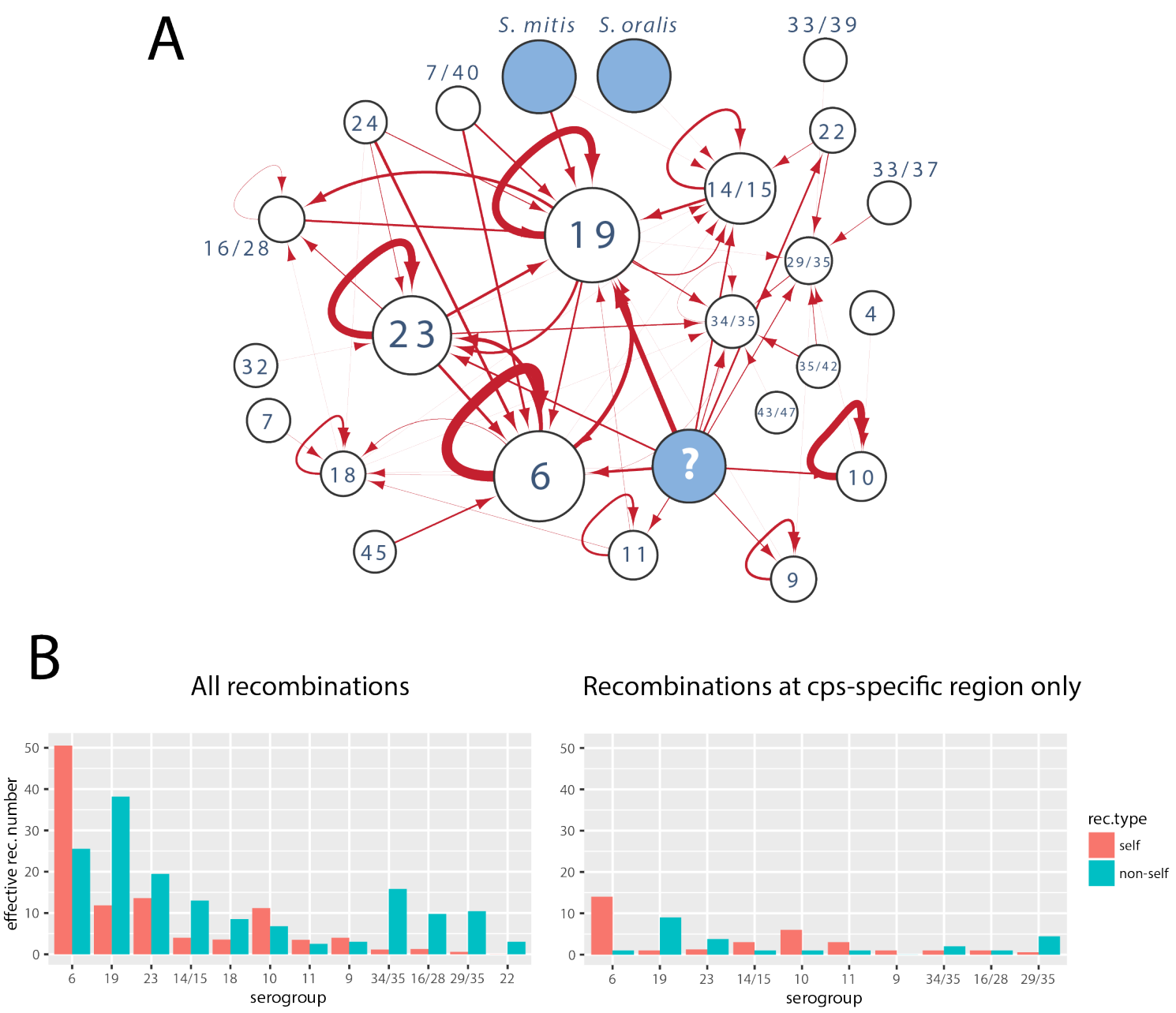

Figure 5: Origin of identified recombinations. (A) The network shows the recombination flow among the serogroups defined in Figure 1A: nodes correspond to serogroups and arrows correspond to the direction of recombination flow based on the most likely origin of the putative recombination events. The width of arrows reflects the number of recombination events (between 0 and 51) and the size of the nodes reflects the number of isolates within the serogroup (except for 'unknown' and other streptococci). (B) Proportion of recombination events originating in the same serogroup (self) versus another serogroup (non-self) for each serogroup. The plot on the left shows all recombinations, while the plot on the right shows recombinations which overlap with the capsule-specific region (minimum 100bp overlap).

\section{Capsular lineage jumping}

Analysis of capsular sequences allows the evolution of the species to be observed from the point of view of the antigen which occasionally alters its genetic background via 'lineage jumping'. Such 
jumps should be observed as alterations of clonal complexes (lineages) within individual clades of serotype trees. The example of serogroup 6 (Fig. 6A) suggests a large within-clade variation of clonal complexes. To estimate the rates of lineage-jumping for four major serogroups $(6,19,23$ and 14/15), we defined a clonal complex (CC) using eBurst (Feil et al., 2004) by linking isolates with 6/7 MLST-locus identity, and a clonal complex group (CCG) by linking isolates with 5/7 MLSTlocus identity. We next used BEAST2 to predict the rate with which an isolate in each serogroup is expected to jump lineage (see Methods). The results are shown in Fig. 6B. We found the mean jumping-rate between CCs to be $4.7 \times 10^{-3}$ jumps per isolate per year, and between CCGs to be $5.0 \times 10^{-4}$ jumps per isolate per year. If changes between all pairs of CCs were equally likely, we would expect that $57 \%$ of them would alter the CCG. Thus, under a random CC-jump model we would expect the CCG jumping rate to be roughly 0.57 times the $\mathrm{CC}$ jumping rate. Instead, we found the CCG jumping rate to be lower than expected. These results suggest that pneumococcal serotypes are less likely to jump lineages if those lineages are very distant. This is consistent with the observation that most pneumococcal serotype switches were previously found to occur within a serogroup (Croucher et al., 2015b). 

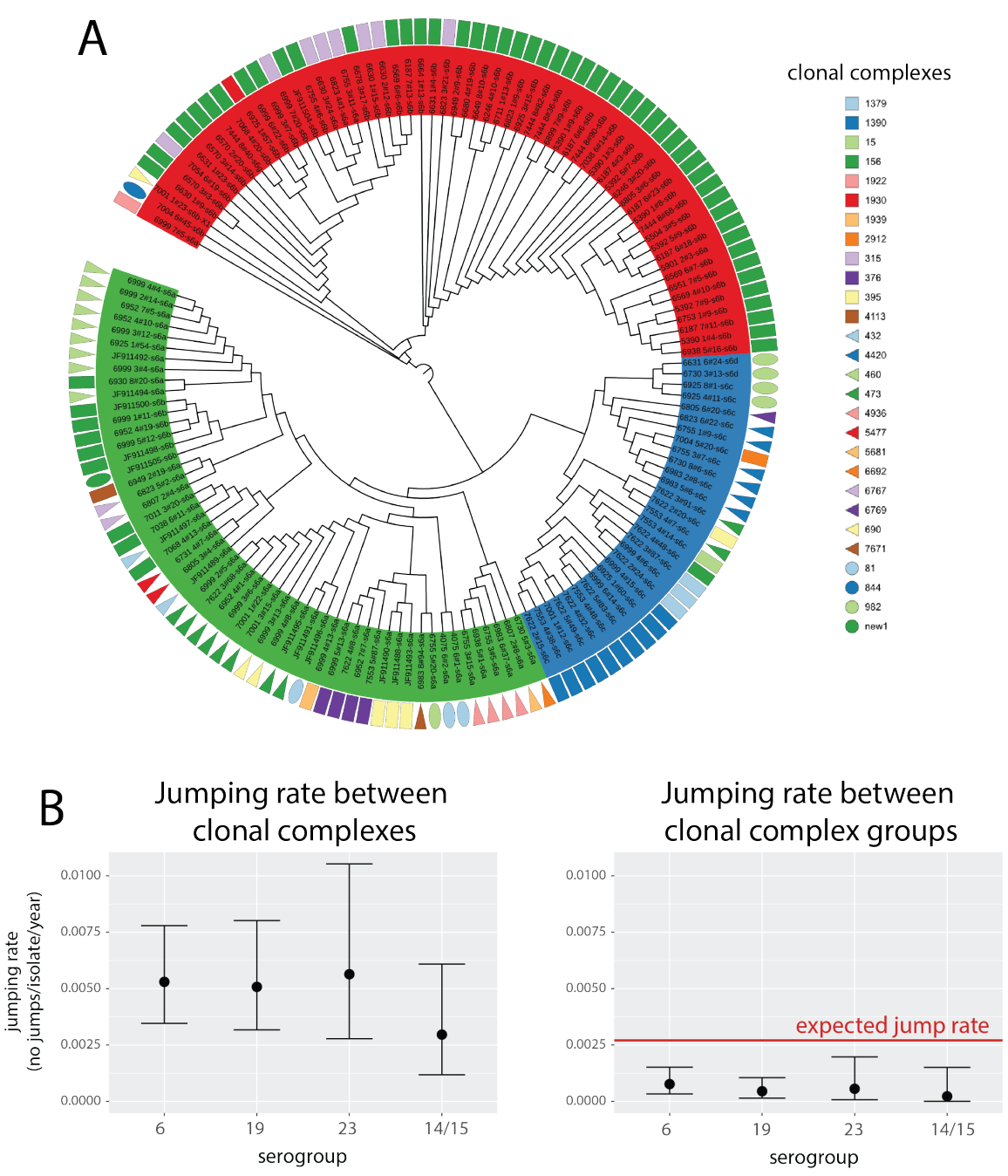

Figure 6: Lineage-jumping dynamics. (A) Dendogram based on the phylogeny of serogroup 6. Tips are grouped according to the three major populations identified: class-I 6A clade (green), 6C/6D clade (blue) and class-II 6B clade (red). Geometric shapes aligned with the tips denote the corresponding clonal complexes of the strains in which the serotype sequence was found. The clades of the tree with branch lengths shorter than $9 \times 10^{-5}$ were collapsed and the most frequent lineage within the clade was plotted. (B) Lineage-jumping rates inferred for the four most frequent serogroups (6, 19, 23 and 14/15) with lineages defined as clonal complexes (CC; left) and as clonal complex groups (CCG; right). The red line shows the CCG jumping rate expected based on the observed CC jumping rate and the assumption that changes between all pairs of CC are equally likely. 


\section{Discussion}

The pneumococcal capsule is an evolutionary hotspot, presumably underlying immune selective pressures acting on this major antigen. In the capsule, we found both elevated molecular clock rate and recombination rate compared to the rest of the genome. The first is consistent with previous observations that non-essential bacterial proteins are expected to evolve faster than essential proteins (Jordan et al., 2002), and non-encapsulated pneumococci have been known to both persist epidemiologically (Chewapreecha et al., 2014) and cause infections (Dixit et al., 2016). We also found the recombination rate at the capsular locus to be around 2.5 times higher than in the rest of the genome (see Fig. S13). The simplest explanation is that this is due to increased recombination-detection power due to increased diversity, however recombination also preferentially occurs between closely related isolates (Majewski et al., 2000; Majewski, 2001; Ansari and Didelot, 2014). Thus, the recombination rate is more likely elevated for the same reasons as the molecular clock, namely that most of observed recombinations are either those which selection has not yet had time to eliminate, or those promoted by diversifying selection. This hypothesis was in line with two observations. First, the average recombination rate was found to be higher in densely sampled Thai collection than in the US collection (see Fig. S12). We think this is because in densely sampled areas there is a higher chance of finding a rare recombination. Second, recent branches of capsular trees had more recombinations than expected from a neutral model (see Fig. S14). Thus, the resulting relaxed purifying selection produces observed recombination hotspots, which translate into an increased diversity over time on which selection can act.

Interestingly, selective pressure can vary between serogroups. The observation that most recombinations in serogroups 19, $2314 / 5$ - but not serogroup 6 - originate in other serogroups is in line with the finding that in former three we found less within-capsule recombinations than expected from a neutral model. One potential explanation is that is that the shorter, within-capsule recombinations are under negative selective pressure to preserve the integrity of the capsular locus. The varying selection pressure across serogroups could be due to different epistatic interactions of capsule-specific genes (glycosyltransferases) as well as also because some serotypes are more likely to recombine with serotypes from the same serogroup (Croucher et al., 2015b). Overall, we can conclude that the observed variation in recombination rates of different serogroups is a combination of microbiological, ecological, epidemiological and evolutionary processes acting on the pneumococcal capsule.

Our results suggest that inter-species recombination plays an important role in the evolution of the cps locus. One prominent example is the emergence of the $19 \mathrm{~B} / 19 \mathrm{C}$ clade by recombination 
of $19 \mathrm{~F}$ with Streptococcus mitis. Furthermore, five detected recombinations bore close resemblance to $S$. mitis and S. oralis isolates. In addition, many glycosyltransferase genes are shared between different species in the mitis group (Kilian et al., 2014). Given the scale of genetic diversity across the mitis group (Kilian et al., 2008), this suggests that many older, hypothesized recombinations within the capsule (for example the emergence of 23A by acquiring wzy gene) were probably acquired from other closely related bacteria. However, the timescales of such process remain unclear and require a better characterisation of the polysaccharide diversity in all nasopharyngeal bacteria.

All evidence thus points at cps being a genetically plastic and dynamic locus, with recombination being its main evolutionary driver. While most recombinations are expected to either be under weak negative selection or produce non-viable capsules (Park et al., 2014), occasionally mosaic, previously unseen capsules can emerge. Indeed, in this study we identified two such mosaic alleles, termed 10X and 21X, while a 33B/33C hybrid was previously identified in an isolate from Denmark (Salter et al., 2012). Given (i) that dense sampling leads to the discovery of more recombinations, (ii) that identifying a novel, mosaic capsule requires a detailed, comparative approach, and (iii) the enormous diversity of glycosyltransferases in the microbial world, we can expect that many more such hybrids are circulating around the world. Why are these hybrids not spreading in the population? This could be due to several different factors, including cross-immunity (Lipsitch, 1997), competitive exclusion (Trzcinski et al., 2015) or fitness differences (Cobey and Lipsitch, 2012). However, introduction of broader, conjugate vaccines in the future may empty ecological niches occupied by the common serotypes, and provide a selective advantage for some of the rare, mosaic serotypes, which could increase in frequency over time. Therefore, a systematic characterisation of capsular diversity across different nasopharyngeal species is important for a better characterisation of the true pneumococcal adaptive potential

\section{Materials \& Methods}

\section{Isolates}

In this study we combined several, previously published genetic and genomic data collections of Streptococcus pneumoniae. These include 3,085 isolates from a continuous mother-infant carriage study in the Mae La refugee camp, Thailand (Turner et al., 2012; Chewapreecha et al., 2014), 616 isolates from children carriage in Massachusetts (Croucher et al., 2013), and 605 isolates from the Pneumococcal Molecular Epidemiology Network of three different lineages: PMEN1 (Croucher et al., 2011), PMEN2 (Croucher et al., 2014b), and PMEN14 (Croucher et al., 2014a). We also 
included 45 sequences of serogroup 6 and 19 isolates from invasive disease from The Netherlands (Elberse et al., 2011), 92 reference sequences (Bentley et al., 2006; Park et al., 2007; Bratcher et al., 2011; Oliver et al., 2013), and a set of 25 reference genomes of $S$. pneumoniae as found in the European Nucleotide Archive www.ebi.ac.uk/genomes/bacteria.html.

\section{Assembling capsular sequences}

All Illumina-sequenced isolates were reassembled using velvet (Zerbino and Birney, 2008) with varied k-mer length (between $50 \%$ and $90 \%$ of the short-read) and the expected coverage (between 5 and 140). The aim was to find an assembly which spanned as much of the entire capsular locus as possible (defined by aligning the assembled and reference sequences using BLASTN with e-value $<10^{-50}$ ) in as few contigs as possible; if multiple such assemblies were produced, the one with the least number of N's and the highest $n_{50}$ value was chosen. Due to the repetitive nature of transposable elements, sequences were assembled without the flanking $\operatorname{dex} B /$ aliA genes and transposable elements. The resulting set of contigs was then analysed for potential misasseblies using reapr (Hunt et al., 2013) and had gaps filled using GapFiller (Nadalin et al., 2012). Finally, isolates with the full capsular locus were scaffolded against the corresponding reference sequence using ABACASS (Assefa et al., 2009) and GapFiller. All poor quality assemblies were removed from the analysis, leaving 3651 capsular allele sequences as well as 162 previously PCR-sequenced isolates. The list of all isolates used in the study is given in Table S1.

\section{Capsular diversity analysis}

To compare the genetic similarity of different reference serotypes, we first collected a list of all proteins located within the capsular locus of all 96 references used as described previously (Bentley et al., 2006; Mavroidi et al., 2007). Of those, we only focused on glycosyltransferases thereby excluding overrepresented $w z g, w z h, w z d$, wze, dexB, aliA, aliB, sugar synthesis genes and transposable elements, giving altogether 742 proteins. All protein sequences were then classified into homology groups with a similar approach to (Mavroidi et al., 2007). Specifically, all-versus-all blastp was run with e-value threshold of $10^{-50}$ with hits with less than $60 \%$ query coverage ignored. The resulting undirected network was analysed with MCL (Enright et al., 2002) with inflation value of 2, and the resulting 254 homology groups were identified. A sequence similarity network was constructed using 96 reference serotypes with nodes representing reference isolates and edges representing similar sequences. Similarity was defined as the proportion of shared homologies between any pair; if the score was asymmetric the higher similarity index was taken. A network was built for a given 
similarity threshold meaning that all pairs above a chosen similarity threshold were connected with an edge. A conservative similarity index of 0.58 produced 40 clusters which were used as a basis for defining a genetic similarity group. Using such clustering, we identified 12 groups which had at least 40 isolates and 500 single nucleotide polymorphisms in the alignment (as given in main text). With the exception of serogroup 19, these genetic similarity groups were identical when defined on the basis significance of shared similarity with threshold of $e=0.01$ based on the approach used by Lima-Mendez et al. (2008).

\section{Obtaining clonal trees of serogroups}

Genetic serogroups were initially aligned using progressiveMauve (Darling et al., 2010). The homologous blocks, identified by the command blastn -task megablast, were aligned using mafft with -ginsi option (Katoh et al., 2002), while the non-homologous blocks were concatenated to avoid force-alignment. All blocks were then concatenated to produce the full alignment. To infer the population genetic structure of serogroups, the Structure software with linkage model was used (Falush et al., 2003). The runs were based on at least 600,000 iterations plus 200,000 burn-in and with multiple chains to insure that the model has converged. The number of populations $\mathrm{K}$ was found as the smallest value of $\mathrm{K}$ which explained the observed population structure, for which

independent runs gave the same output, and which was supported by the value obtained by BAPS (Corander and Marttinen, 2006). In all examined cases, the identified populations corresponded well to major serotypes or serotype groups (see Text S1 for the results of the population structure analyses). Between-population recombinations were defined by Structure as those with the minimum posterior probability for originating in a different population of 0.75 and reaching at least 0.95 at one site, and removed. The initial phylogeny was generated using PhyML (Guindon et al., 2010). Next, each population was analysed by Gubbins (Croucher et al., 2015a) with the initial phylogeny used as a starting tree. Recombinations identified by Gubbins or Structure were removed. The resulting clonal alignments were then analysed again by Structure to identify potential hierarchical population structure and within-population recombinations which Gubbins could not detect. In the final alignment, all regions identified by Structure or Gubbins were removed from the alignment to generate the final phylogeny. The pattern of recombinations on this tree was predicted by both Gubbins (running a single iteration conditional on the final phylogeny with two window sizes: $1 \mathrm{~kb}$ and 10kb) and Structure (using the ace() function in ape package in R to predict the most likely ancestral pattern (Paradis et al., 2004)). The two types of recombinations were then merged into the final list of recombinations with overlapping blocks merged, however because Gubbins has a more 
elaborate algorithm of predicting ancestral recombinations based on ancestral SNP reconstruction, Structure-recombinations at internal nodes which did not overlap with Gubbins-recombinations were ignored. All recombinations ancestral to each of the $\mathrm{K}$ populations found were ignored due to low detection power of events on long tree branches.

\section{Estimating timescales using BEAST}

To estimate the molecular clock of the capsular locus, three collections of pneumococcal wholegenomes were used, PMEN1, PMEN2 and PMEN14 (Croucher et al., 2011, 2014b,a). Each collection was assembled, aligned and recombinations were removed using Gubbins (Croucher et al., 2015a), with details of the processing analysis given in the original publications. The alignment was then divided into the capsule-only alignment (defined by the starting position of the $\operatorname{dex} B$ and the ending position of the aliA gene) and the clonal alignment with the capsule removed. The two alignments were then analysed using BEAST2 (Bouckaert et al., 2014) using a single analysis with parameters shared between alignments. In particular, we assumed the same substitution model (GTR with four gamma categories), the same tree prior (Coalescent Bayesian Skyline), and tree but different parameters of the clock model (Relaxed Clock Log Normal). We ensured that all parameters were estimated with effective sample size (ESS) above 200. The results of the ucldMean parameter for the capsule were then pooled together to which a log normal distribution was fit, yielding best fit parameter of $\mu=-12.98$. Next, the clonal alignments for each serogroup (i.e., with recombinations removed) were analysed using BEAST2, and the same set of models was fit as in the case of whole genomes with three exceptions. First, we assumed a coalescent constant population tree prior. Second, an informative prior for the molecular (strict) clock was used with the value of $\mu$ estimated using whole-genomes. Third, the phylogeny was fixed as the final clonal phylogeny obtained in the previous section, thereby estimating divergence times and the dates for the recombination events assigned to each node of the tree. A similar approach was considered to estimate the lineage-jumping rate. Lineages were identified as clonal complexes (CCs) and clonal complex groups (CCGs; see main text). These lineages were next treated as discrete traits to perform a discrete trait phylogenetic analysis (Lemey et al., 2009) by modelling switches of the genomic lineage background as substitutions in the discrete trait. Sampling of isolates was blind to the genomic background, so in this context no sampling bias for the discrete trait analysis was expected (De Maio et al., 2015). Each sub-population was considered as a separate phylogeny with the shared genetic clock and the jump-rate shared between phylogenies within the same serogroup. Due to simplicity a homogeneous jumping-rate was assumed. 


\section{Estimation of recombination rates}

In order to estimate the recombination rate for each serogroup, we fitted a basic model describing the distribution of recombination events on a tree using a Poisson process; see also Mostowy et al. (2014). The number of recombinations at each branch of the tree was modelled as a Poissondistributed random variable $m_{i}$ with mean $\lambda L_{i}$, where $\lambda$ is the inferred recombination rate and $L_{i}$ is the branch length in years. The estimated recombination rate $\lambda$ was the value which maximised the likelihood $\ln \prod_{i=1}^{B}$ Pois $\left(m_{i} ; \lambda L_{i}\right)$, where $B$ is the number of tree branches. A single recombination rate was estimated for each serogroup. To avoid estimate bias we excluded all long branches of the tree, namely branches leading to the most recent common ancestors for each subpopulation (of the K populations estimated by Structure) together with all their ancestor branches.

To estimate recombination rate derived from whole genome data, we used clonal trees obtained as described in the original publications for each of the whole-genome collection used in this work. Briefly, for each bacterial lineage, a clonal tree was estimated with recombinations aligned against nodes of the tree. Genes $\operatorname{dex} B$ and aliA were used to identify the capsule coordinates and distinguish background- from capsule-recombinations. Lineages of predominantly the same serogroup (minimum 95\%) were used collectively to estimate the mean recombination rate by combining the information about the number of recombination events on each branch and the corresponding branch length in units of years, and fitting the Poisson model described above. The lineages used to estimate the recombination rates the four analysed serogroups were as follows (please refer to original publications for details). Serogroup 6: Massachusetts (MA) lineages 10, 13 and 14 and PMEN2 (altogether 247 isolates); serogroup 19: MA lineage 15, MaeLa 1 and PMEN14 (altogether 564 isolates); serogroup 23: MA lineage 9 and PMEN1 (altogether 297 isolates); serogroup 14/15: MA lineage 3 and MaeLa lineage 7 (altogether 126 isolates; remaining MaeLa lineages were ignored due to the absence of the full resolution capsular locus in the original alignment).

\section{Acknowledgements}

This work was supported by the EU Marie Skłodowska-Curie Intra-European fellowship (project no. 329515, R-EVOLUTION PNEUMO to R.J.M.), Junior Research Fellowship from Imperial College London (to R.J.M.), Sir Henry Wellcome Fellowship (107376/Z/15/Z to C.C.), NIH MIDAS program (grant U01GM110721 to C.F.) and The Wellcome Trust (grant no. 09805). The authors

thank Marc Lipsitch and Bill Hanage for interesting comments and discussions, James McInerney for help with network analyses, Remco Bouckaert and Denise Kühnert for help with BEAST anal- 
yses, Aleksandra Królik for help with artwork and Statens Serum Institut for the serotyping work on three $21 \mathrm{X}$ isolates.

\section{References}

Aanensen, D. M., Mavroidi, A., Bentley, S. D., Reeves, P. R., and Spratt, B. G. 2007. Predicted functions and linkage specificities of the products of the Streptococcus pneumoniae capsular biosynthetic loci. J. Bacteriol., 189(21): 7856-7876.

Ansari, M. A. and Didelot, X. 2014. Inference of the properties of the recombination process from whole bacterial genomes. Genetics, 196(1): 253-265.

Assefa, S., Keane, T. M., Otto, T. D., Newbold, C., and Berriman, M. 2009. ABACAS: algorithmbased automatic contiguation of assembled sequences. Bioinformatics, 25(15): 1968-1969.

Bapteste, E., Lopez, P., Bouchard, F., Baquero, F., McInerney, J. O., and Burian, R. M. 2012. Evolutionary analyses of non-genealogical bonds produced by introgressive descent. Proc. Natl. Acad. Sci. U.S.A., 109(45): 18266-18272.

Bentley, S. D., Aanensen, D. M., Mavroidi, A., Saunders, D., Rabbinowitsch, E., Collins, M., Donohoe, K., Harris, D., Murphy, L., Quail, M. A., Samuel, G., Skovsted, I. C., Kaltoft, M. S., Barrell, B., Reeves, P. R., Parkhill, J., and Spratt, B. G. 2006. Genetic analysis of the capsular biosynthetic locus from all 90 pneumococcal serotypes. PLoS Genet., 2(3): e31.

Bouckaert, R., Heled, J., Kuhnert, D., Vaughan, T., Wu, C. H., Xie, D., Suchard, M. A., Rambaut, A., and Drummond, A. J. 2014. BEAST 2: a software platform for Bayesian evolutionary analysis. PLoS Comput. Biol., 10(4): e1003537.

Bratcher, P. E., Park, I. H., Oliver, M. B., Hortal, M., Camilli, R., Hollingshead, S. K., Camou, T., and Nahm, M. H. 2011. Evolution of the capsular gene locus of Streptococcus pneumoniae serogroup 6. Microbiology (Reading, Engl.), 157(Pt 1): 189-198.

Briles, D. E., Crain, M. J., Gray, B. M., Forman, C., and Yother, J. 1992. Strong association between capsular type and virulence for mice among human isolates of Streptococcus pneumoniae. Infect. Immun., 60(1): 111-116.

Bruen, T. C., Philippe, H., and Bryant, D. 2006. A simple and robust statistical test for detecting the presence of recombination. Genetics, 172(4): 2665-2681. 
Chewapreecha, C., Harris, S. R., Croucher, N. J., Turner, C., Marttinen, P., Cheng, L., Pessia, A., Aanensen, D. M., Mather, A. E., Page, A. J., Salter, S. J., Harris, D., Nosten, F., Goldblatt, D., Corander, J., Parkhill, J., Turner, P., and Bentley, S. D. 2014. Dense genomic sampling identifies highways of pneumococcal recombination. Nat. Genet., 46(3): 305-309.

Cobey, S. and Lipsitch, M. 2012. Niche and neutral effects of acquired immunity permit coexistence of pneumococcal serotypes. Science, 335(6074): 1376-1380.

Corander, J. and Marttinen, P. 2006. Bayesian identification of admixture events using multilocus molecular markers. Mol. Ecol., 15(10): 2833-2843.

Croucher, N. J., Harris, S. R., Fraser, C., Quail, M. A., Burton, J., van der Linden, M., McGee, L., von Gottberg, A., Song, J. H., Ko, K. S., Pichon, B., Baker, S., Parry, C. M., Lambertsen, L. M., Shahinas, D., Pillai, D. R., Mitchell, T. J., Dougan, G., Tomasz, A., Klugman, K. P., Parkhill, J., Hanage, W. P., and Bentley, S. D. 2011. Rapid pneumococcal evolution in response to clinical interventions. Science, 331(6016): 430-434.

Croucher, N. J., Finkelstein, J. A., Pelton, S. I., Mitchell, P. K., Lee, G. M., Parkhill, J., Bentley, S. D., Hanage, W. P., and Lipsitch, M. 2013. Population genomics of post-vaccine changes in pneumococcal epidemiology. Nat. Genet., 45(6): 656-663.

Croucher, N. J., Chewapreecha, C., Hanage, W. P., Harris, S. R., McGee, L., van der Linden, M., Song, J. H., Ko, K. S., de Lencastre, H., Turner, C., Yang, F., Sa-Leao, R., Beall, B., Klugman, K. P., Parkhill, J., Turner, P., and Bentley, S. D. 2014a. Evidence for soft selective sweeps in the evolution of pneumococcal multidrug resistance and vaccine escape. Genome Biol Evol, 6(7): 1589-1602.

Croucher, N. J., Hanage, W. P., Harris, S. R., McGee, L., van der Linden, M., de Lencastre, H., Sa-Leao, R., Song, J. H., Ko, K. S., Beall, B., Klugman, K. P., Parkhill, J., Tomasz, A., Kristinsson, K. G., and Bentley, S. D. 2014b. Variable recombination dynamics during the emergence, transmission and 'disarming' of a multidrug-resistant pneumococcal clone. $B M C$ Biol., 12: 49.

Croucher, N. J., Page, A. J., Connor, T. R., Delaney, A. J., Keane, J. A., Bentley, S. D., Parkhill, J., and Harris, S. R. 2015a. Rapid phylogenetic analysis of large samples of recombinant bacterial whole genome sequences using Gubbins. Nucleic Acids Res., 43(3): e15. 
Croucher, N. J., Kagedan, L., Thompson, C. M., Parkhill, J., Bentley, S. D., Finkelstein, J. A., Lipsitch, M., and Hanage, W. P. 2015b. Selective and genetic constraints on pneumococcal serotype switching. PLoS Genet., 11(3): e1005095.

Darling, A. E., Mau, B., and Perna, N. T. 2010. progressiveMauve: multiple genome alignment with gene gain, loss and rearrangement. PLoS ONE, 5(6): e11147.

De Maio, N., Wu, C. H., O’Reilly, K. M., and Wilson, D. 2015. New Routes to Phylogeography: A Bayesian Structured Coalescent Approximation. PLoS Genet., 11(8): e1005421.

Dixit, C., Keller, L. E., Bradshaw, J. L., Robinson, D. A., Swiatlo, E., and McDaniel, L. S. 2016. Nonencapsulated Streptococcus pneumoniae as a cause of chronic adenoiditis. IDCases, 4: 56-58.

Elberse, K., Witteveen, S., van der Heide, H., van de Pol, I., Schot, C., van der Ende, A., Berbers, G., and Schouls, L. 2011. Sequence diversity within the capsular genes of Streptococcus pneumoniae serogroup 6 and 19. PLoS ONE, 6(9): e25018.

Enright, A. J., Van Dongen, S., and Ouzounis, C. A. 2002. An efficient algorithm for large-scale detection of protein families. Nucleic Acids Res., 30(7): 1575-1584.

Falush, D., Stephens, M., and Pritchard, J. K. 2003. Inference of population structure using multilocus genotype data: linked loci and correlated allele frequencies. Genetics, 164(4): 15671587.

Feil, E. J., Smith, J. M., Enright, M. C., and Spratt, B. G. 2000. Estimating recombinational parameters in Streptococcus pneumoniae from multilocus sequence typing data. Genetics, 154(4): 1439-1450.

Feil, E. J., Li, B. C., Aanensen, D. M., Hanage, W. P., and Spratt, B. G. 2004. eBURST: inferring patterns of evolutionary descent among clusters of related bacterial genotypes from multilocus sequence typing data. J. Bacteriol., 186(5): 1518-1530.

Geno, K. A., Gilbert, G. L., Song, J. Y., Skovsted, I. C., Klugman, K. P., Jones, C., Konradsen, H. B., and Nahm, M. H. 2015. Pneumococcal Capsules and Their Types: Past, Present, and Future. Clin. Microbiol. Rev., 28(3): 871-899.

Guindon, S., Dufayard, J. F., Lefort, V., Anisimova, M., Hordijk, W., and Gascuel, O. 2010. New algorithms and methods to estimate maximum-likelihood phylogenies: assessing the performance of PhyML 3.0. Syst. Biol., 59(3): 307-321. 
Henrichsen, J. 1995. Six newly recognized types of Streptococcus pneumoniae. J. Clin. Microbiol., 33(10): 2759-2762.

Henriques-Normark, B., Blomberg, C., Dagerhamn, J., Battig, P., and Normark, S. 2008. The rise and fall of bacterial clones: Streptococcus pneumoniae. Nat. Rev. Microbiol., 6(11): 827-837.

Hunt, M., Kikuchi, T., Sanders, M., Newbold, C., Berriman, M., and Otto, T. D. 2013. REAPR: a universal tool for genome assembly evaluation. Genome Biol., 14(5): R47.

Hyams, C., Camberlein, E., Cohen, J. M., Bax, K., and Brown, J. S. 2010. The Streptococcus pneumoniae capsule inhibits complement activity and neutrophil phagocytosis by multiple mechanisms. Infect. Immun., 78(2): 704-715.

Jordan, I. K., Rogozin, I. B., Wolf, Y. I., and Koonin, E. V. 2002. Essential genes are more evolutionarily conserved than are nonessential genes in bacteria. Genome Res., 12(6): 962-968.

Kadioglu, A., Weiser, J. N., Paton, J. C., and Andrew, P. W. 2008. The role of Streptococcus pneumoniae virulence factors in host respiratory colonization and disease. Nat. Rev. Microbiol., 6(4): 288-301.

Katoh, K., Misawa, K., Kuma, K., and Miyata, T. 2002. MAFFT: a novel method for rapid multiple sequence alignment based on fast Fourier transform. Nucleic Acids Res., 30(14): 3059-3066.

Kilian, M., Poulsen, K., Blomqvist, T., Havarstein, L. S., Bek-Thomsen, M., Tettelin, H., and Sørensen, U. B. 2008. Evolution of Streptococcus pneumoniae and its close commensal relatives. PLoS ONE, 3(7): e2683.

Kilian, M., Riley, D. R., Jensen, A., Bruggemann, H., and Tettelin, H. 2014. Parallel evolution of Streptococcus pneumoniae and Streptococcus mitis to pathogenic and mutualistic lifestyles. MBio, 5(4): e01490-01414.

Lemey, P., Rambaut, A., Drummond, A. J., and Suchard, M. A. 2009. Bayesian phylogeography finds its roots. PLoS Comput. Biol., 5(9): e1000520.

Lima-Mendez, G., Van Helden, J., Toussaint, A., and Leplae, R. 2008. Reticulate representation of evolutionary and functional relationships between phage genomes. Mol. Biol. Evol., 25(4): 762-777. 
Lipsitch, M. 1997. Vaccination against colonizing bacteria with multiple serotypes. Proc. Natl. Acad. Sci. U.S.A., 94(12): 6571-6576.

Majewski, J. 2001. Sexual isolation in bacteria. FEMS Microbiol. Lett., 199(2): 161-169.

Majewski, J., Zawadzki, P., Pickerill, P., Cohan, F. M., and Dowson, C. G. 2000. Barriers to genetic exchange between bacterial species: Streptococcus pneumoniae transformation. J. Bacteriol., 182(4): 1016-1023.

Mavroidi, A., Aanensen, D. M., Godoy, D., Skovsted, I. C., Kaltoft, M. S., Reeves, P. R., Bentley, S. D., and Spratt, B. G. 2007. Genetic relatedness of the Streptococcus pneumoniae capsular biosynthetic loci. J. Bacteriol., 189(21): 7841-7855.

Morona, J. K., Miller, D. C., Morona, R., and Paton, J. C. 2004. The effect that mutations in the conserved capsular polysaccharide biosynthesis genes cpsA, cpsB, and cpsD have on virulence of Streptococcus pneumoniae. J. Infect. Dis., 189(10): 1905-1913.

Mostowy, R., Croucher, N. J., Hanage, W. P., Harris, S. R., Bentley, S., and Fraser, C. 2014. Heterogeneity in the frequency and characteristics of homologous recombination in pneumococcal evolution. PLoS Genet., 10(5): e1004300.

Nadalin, F., Vezzi, F., and Policriti, A. 2012. GapFiller: a de novo assembly approach to fill the gap within paired reads. BMC Bioinformatics, 13 Suppl 14: S8.

Oliver, M. B., van der Linden, M. P., Kuntzel, S. A., Saad, J. S., and Nahm, M. H. 2013. Discovery of Streptococcus pneumoniae serotype 6 variants with glycosyltransferases synthesizing two differing repeating units. J. Biol. Chem., 288(36): 25976-25985.

Paradis, E., Claude, J., and Strimmer, K. 2004. APE: analyses of phylogenetics and evolution in R language. Bioinformatics, 20: 289-290.

Park, I. H., Park, S., Hollingshead, S. K., and Nahm, M. H. 2007. Genetic basis for the new pneumococcal serotype, 6C. Infect. Immun., 75(9): 4482-4489.

Park, I. H., Geno, K. A., Sherwood, L. K., Nahm, M. H., and Beall, B. 2014. Population-based analysis of invasive nontypeable pneumococci reveals that most have defective capsule synthesis genes. PLoS ONE, 9(5): e97825. 
Salter, S. J., Hinds, J., Gould, K. A., Lambertsen, L., Hanage, W. P., Antonio, M., Turner, P., Hermans, P. W., Bootsma, H. J., O'Brien, K. L., and Bentley, S. D. 2012. Variation at the capsule locus, cps, of mistyped and non-typable Streptococcus pneumoniae isolates. Microbiology (Reading, Engl.), 158(Pt 6): 1560-1569.

Schaffner, T. O., Hinds, J., Gould, K. A., Wuthrich, D., Bruggmann, R., Kuffer, M., Muhlemann, K., Hilty, M., and Hathaway, L. J. 2014. A point mutation in cpsE renders Streptococcus pneumoniae nonencapsulated and enhances its growth, adherence and competence. BMC Microbiol., 14: 210 .

Trzcinski, K., Li, Y., Weinberger, D. M., Thompson, C. M., Cordy, D., Bessolo, A., Malley, R., and Lipsitch, M. 2015. Effect of Serotype on Pneumococcal Competition in a Mouse Colonization Model. MBio, 6(5): e00902-00915.

Turner, P., Turner, C., Jankhot, A., Helen, N., Lee, S. J., Day, N. P., White, N. J., Nosten, F., and Goldblatt, D. 2012. A longitudinal study of Streptococcus pneumoniae carriage in a cohort of infants and their mothers on the Thailand-Myanmar border. PLoS ONE, 7(5): e38271.

van Tonder, A. L., Bray, J. E., Quirk, S. J., Haraldsson, G., Jolley, K. A., Maiden, M. C., Hoffmann, S., Bentley, S. D., Haraldsson, Á., Erlendsdóttir, H., Kristinsson, K. G., and Brueggemann, A. B. 2016. Putatively novel serotypes and the potential for reduced vaccine effectiveness: capsular locus diversity revealed among 5,405 pneumococcal genomes. Microbial Genomics. Accepted for publication.

Vos, M. and Didelot, X. 2009. A comparison of homologous recombination rates in bacteria and archaea. ISME J, 3(2): 199-208.

Weinberger, D. M., Trzcinski, K., Lu, Y. J., Bogaert, D., Brandes, A., Galagan, J., Anderson, P. W., Malley, R., and Lipsitch, M. 2009. Pneumococcal capsular polysaccharide structure predicts serotype prevalence. PLoS Pathog., 5(6): e1000476.

WHO 2012. Estimated Hib and Pneumococcal Deaths for Children Under 5 Years of Age, 2008. Technical report, World Health Organization, Immunization, Vaccines and Biologicals.

Yang, Z. 2007. PAML 4: phylogenetic analysis by maximum likelihood. Mol. Biol. Evol., 24(8): 1586-1591. 
Yother, J. 2011. Capsules of Streptococcus pneumoniae and other bacteria: paradigms for polysaccharide biosynthesis and regulation. Annu. Rev. Microbiol., 65: 563-581.

Zerbino, D. R. and Birney, E. 2008. Velvet: algorithms for de novo short read assembly using de Bruijn graphs. Genome Res., 18(5): 821-829. 